\title{
Représentations du théâtre russe dans la presse francophone des Lumières
}

\author{
Carole Chapin \\ Université Paris 3 Sorbonne Nouvelle \\ carolechapin@gmail.com
}

\begin{abstract}
In the eighteenth century, journals carried information and promoted literary productions. They also acted as conduits for the reception of foreign cultures: thus, they show the ambiguities of Franco-Russian cultural relations. They acted as a medium that facilitated debates and polemics. This phenomenon is particularly interesting when the journalists discussed theatre, because this art form can act as a political and social instrument. Interest in the Russian theatre in French-speaking periodicals in the eighteenth century was not only a sign of greater interest in 'Russian subjects', but also formed part of a general reflection about contemporary drama. This trend was characterized by more openness towards foreign cultures. During this period of theoretical questioning, national theatre cultures tended to strengthen their specificities, while also using borrowings and translations from others theatres. The interest of French theatre aficionados for Russian theatre is well-known, as is the enthusiasm of the purveyors of Russian theatre for French theatre. However, the choice of words used to describe Russian theatre in the Francophone press-whether they be of praise or of criticism-can hide other goals, such as showing the greatness of Catherine II's government or insisting on the role of the French model in Russia's cultural development.

In this paper, a corpus of selected articles is used to show several practices and issues o pertaining to the topic of the Russian theatre in the Francophone press. We question several possible political and aesthetic consequences of the image of Russia, which were spread by French-speaking periodicals in Europe. Furthermore, we try to analyze the role played by the specific discourse used by the periodicals in order to demonstrate its importance for researchers working on eighteenth-century European theatre and cultural relations.
\end{abstract}

Mots clé

théâtre ; Russie ; XVIII ${ }^{\mathrm{e}}$ siècle ; périodiques ; critique ; politique culturelle

Le journal est un «théâtre ouvert », s'enthousiasme Prévost dans son « Avertissement » du Journal Étranger, en $1755^{1}$. La nécessité, soulignée par ce journal, de regarder vers l'étranger pour améliorer la littérature nationale, n'est pas une originalité française mais un mouvement général en Europe au XVIII ${ }^{\mathrm{e}}$ siècle. Un mouvement dans lequel la presse joue un rôle fondamental : « Les étrangers auront la liberté de m'envoyer leurs ouvrages. Ils trouveront un accueil plein de reconnaissance, pour leurs remarques, leurs extraits et leurs moindres fragments $^{2}$ », ajoute-t-il. Ce mouvement n'en est pas moins ambigu : il recouvre souvent aussi une volonté de construire, d'améliorer ou de représenter les œuvres nationales positivement.

La presse russe, tournée vers l'Europe, est un cas exemplaire de l'intérêt pour l'autre dans la construction de soi. La période que nous allons aborder ici, de la fin des années 1750 au début des années 1780 , correspond à une étape d'un processus de développement des lettres russes. Depuis la fin du XVII ${ }^{\mathrm{e}}$ siècle, et notamment suite à la fondation de Saint-Pétersbourg, la

\footnotetext{
${ }^{1}$ Abbé Prévost, « Avertissement de M. l'abbé Prévost sur la continuation du Journal Étranger », Journal étranger, Paris et Bruxelles, Durant, janvier 1755.

${ }^{2}$ Loc.cit.
} 
Russie est entrée dans une période de rapprochement et d'un apprentissage accéléré vis-à-vis de la culture européenne. L'initiative de ce rapprochement appartient au monarque et à ses proches collaborateurs. La connaissance des langues étrangères s'étend : le français devient la langue des interactions de la noblesse ainsi que celle de nombreux divertissements comme le théâtre ${ }^{3}$. Aussi donne-t-elle accès aux classiques étrangers, et les journaux russes soutiennent ces désirs de l'élite lettrée, tout en faisant la promotion du développement de la littérature nationale. En effet, la dynamique de développement de la presse en Russie est corrélée aux enjeux éducatifs, en particulier à l'éducation de la noblesse. Les premiers journaux, imprimés au début du siècle, sont nés du désir de Pierre le Grand de doter la Russie d'un organe d'information, mais ceux-ci restent assez peu nombreux jusqu'à la fin des années 1750 . Toutefois à partir de cette période on constate une augmentation significative du nombre des journaux, dont un nombre important portent des titres qui insistent sur leur dimension morale et didactique : par exemple Prazdnoé Vremia ${ }^{4}$ (1759), Poleznoé Uvesselenié5 (1760), Nevinnoe Ouprajnenié ${ }^{6}$ (1763) et Dobroé Namerenie ${ }^{7}$ (1764). Ces appellations se démarquent par la mise en valeur de la lecture du journal en tant que plaisir et divertissement, ce qui les distingue et les oppose, comme le leur reprochent leurs détracteurs, aux textes dits " sérieux ». Mais le divertissement n'est jamais superflu et superficiel, il soutient le projet didactique car il provoque une lecture active, une implication du lecteur et un dialogue avec ce dernier. Ce divertissement utile exerce une influence positive sur l'homme et la société dans laquelle il vit, et permet, par extension, d'appliquer son esprit critique aux autres divertissements, comme le théâtre. A partir de 1769, date de la publication de Vsiakaia $V_{\text {siatchina }}{ }^{8}$, l'évolution du nombre de publications est encore plus saisissante : les années 1770 virent ainsi l'apparition d'une vingtaine de périodiques, soit le double des décennies précédentes. On trouve alors un nombre important de traductions, d'adaptations et de reprises des journaux moralistes anglais et français, avec ou sans mention des sources. Audelà du rapport à l'étranger, c'est la construction de la presse russe, de son ton original, de la formation de ses lecteurs qui est en jeu, ainsi que les conséquences qui en découlent. Il n’est alors pas étonnant que ces périodiques soient à la fois très liés à des institutions de l'État et au projet politique de Catherine II, et dans le même temps l'espace pour développer opinions contraires et esprit critique, y compris à l'égard de ces mêmes institutions. Rappelons que l'impulsion pour le développement de ce type de périodiques ${ }^{9}$ vient de l'Impératrice ellemême, rédactrice officieuse de Vsiakaia Vsiatchina publié sous le nom de son secrétaire Grigory Kozitsky, et que ce périodique ainsi que beaucoup de journaux importants de la

\footnotetext{
${ }^{3}$ Voir l'avant-propos et l'introduction du dictionnaire Les Français en Russie au siècle des Lumières. Anne Mézin, et Vladislav Rjéoutski (dir.), Les Français en Russie au siècle des Lumières : dictionnaire des Français, Suisses, Wallons et autres francophones en Russie de Pierre le Grand à Paul I ${ }^{e r}$, Ferney-Voltaire : Centre international d'étude du XVIII siècle, Paris, Amateurs de livres, 2011, pp. xix-xx et 3-4. Alexeï Evstratov, Le théâtre francophone à Saint-Pétersbourg sous le règne de Catherine II (1762-1796). Organisation, circulation et symboliques des spectacles dramatiques. Thèse de doctorat, [Paris,] Université Paris-Sorbonne (Paris IV), 2012 ; et Derek Offord, Lara Ryazanova-Clarke, Vladislav Rjéoutski et Gesine Argent (dir.), French and Russian in Imperial Russia, Edimbourg, Edinburgh University Press, 2015, 2 vol.

${ }^{4}$ Piotr Pastoukhov (rédacteur à partir de 176o), Праздное время в пользу употребленное [Le Temps libre bien utilisé], Saint-Pétersbourg, 1759-176o.

${ }^{5}$ Mikhail Kheraskov, Полезное увеселение [Le Divertissement utile], Moscou, Moskovski ouniversitet, 1760-1762.

${ }^{6}$ Hyppolite Bogdanovitch, Невинное упражнение [L'Exercice innocent], Moscou, 1763.

${ }^{7}$ Vassily Sankovsky, Доброе намерение [La Bonne volonté], Moscou, 1764.

${ }^{8}$ Grigory Kozitski (red.), Всякая всячина [Pot-pourri], Saint-Pétersbourg, 1769-1770.

${ }^{9}$ Appelés «périodiques satiriques » (1769 à 1774). Voir par exemple la classification de Павел Берков, История русской журналистики XVIII века [Pavel Berkov, Histoire du journalisme russe au XVIII ${ }^{e}$ siècle], Moscou, Leningrad, Izdatelstvo Akademii Naouk SSSR, 1952, p. 156-224.
} 
période comme Prazdnoé Vremia v polzou oupotreblennoe (Le Temps libre bien utilisé) ${ }^{10}$, Poleznoé Uvesselenié (Le Divertissement utile) ${ }^{11}$, Dobroé Namerenie (La Bonne Volonté) ${ }^{12}$, ou encore Poleznoé s priatnym (L'Utile et l'agréable) ${ }^{13}$ sont édités par des organes officiels comme l'Académie des Sciences, l'Université de Moscou ou le Corps des Cadets de Saint-Pétersbourg. Il s'agit véritablement d'un projet éducatif qui forme à la lecture par la lecture, et propose des modèles. Pour le servir, la satire souhaitée ne doit pas être une critique acerbe et un rire moqueur, mais une critique douce qui corrige par l'humour sans blesser violemment l'adversaire. Cependant cette formation et l'implication du lecteur permet également un décalage par rapport aux règles et aux modèles, sujet de débat et outil du débat. C'est ainsi que les discours qui sous-tendent le développement de la presse russe, ainsi qu'en témoignent préfaces et commentaires métatextuels, défendent son rôle dans l'éducation des lecteurs : les journaux ont pour vocation d'informer son public des nouveautés littéraires, des événements et des spectacles. L'enjeu national se joint à ce désir pédagogique : il n'est pas seulement nécessaire d'informer mais aussi de transformer la perception, des lecteurs russes ainsi que de lecteurs étrangers pour qui les journaux russes sont une source d'informations. Il s'agit de communiquer le plus largement possible l'image d'une Russie éclairée, et de stimuler encore davantage, grâce à elle, le développement des arts et des lettres et l'intérêt des spectateurs et des lecteurs.

L'éducation des élites russes modifie leur rapport aux littératures étrangères et leur permet de se situer sur le même plan culturel que leurs homologues européennes, mais cette réalité doit également être diffusée afin de permettre une modification profonde de l'image de la Russie. La cour russe a tout intérêt à être reconnue comme une cour éclairée qui encourage le développement des sciences et des arts en Russie. Là encore, la presse joue un rôle fondamental: cette image circule grâce à des stratégies personnelles, aux réseaux des journalistes et littérateurs, mais aussi par l'intermédiaire de stratégies politiques, par les réseaux des diplomates, sur lesquels nous devrons nous pencher pour éclairer quelques exemples.

Quelle est la réception de cette stratégie ? Les extraits dont nous disposons permettent de s'interroger sur les réactions des journalistes, et, par leur intermédiaire, sur les informations transmises aux lecteurs des périodiques francophones européens. L'étude de la presse francophone, à ce titre, ne laisse pas d'être complexe, car les débats sur le théâtre sont influencés par l'image et la réputation des pays. La France bénéficie d'une aura particulière : elle est décrite comme le fer de lance de la culture européenne, le modèle à imiter et le laboratoire des nouveautés, des innovations et des modes, cependant la critique des lettres et des arts étrangers représente une forme de concurrence. Quant à la représentation de la Russie dans la presse francophone, elle est en pleine mutation au cours de la période étudiée. L'image d'une Russie sauvage s'estompe peu à peu mais conserve des permanences : elle n'est pas encore vraiment européenne, quoiqu'en plein développement, ce qui attise la curiosité. Le désir de la cour, représentée par les élites russes, de présenter la Russie et l'avancée de ses sciences et arts en Europe pour modifier son image se traduit ainsi dans les pages des journaux. De nombreux moyens ont été mis en œuvre, l'un d'entre eux étant la demande officielle de la cour d'Elisabeth à Voltaire de rédiger une histoire de la Russie sous Pierre le

\footnotetext{
${ }^{10}$ Piotr Pastoukhov, op.cit.

${ }^{11}$ Mikhail Kheraskov, op.cit.

${ }^{12}$ Vassily Sankovsky, op.cit.

${ }^{13}$ Ivan Roumiantsev et Ignace de Teyls, Полезное с прятным [L'Utile et l'agréable], Saint-Pétersbourg, 1769.
} 
Grand en $1757^{14}$, suivie par des initiatives de Catherine II pour présenter la Russie sous son meilleur jour. Cependant, les stéréotypes d'un « royaume grossier et barbare » et d'une société "d'une infinie brutalité ${ }^{15}$ », sont persistants. La presse européenne contemporaine joue alors un rôle particulièrement important, car un changement de ton - la louange plutôt que la critique - et de sujet - mettre les arts et les lettres au premier plan - est un enjeu majeur. A ce titre, les discours sur le théâtre présentent un intérêt particulier, car le développement de l'art dramatique est perçu comme un signe indéniable du développement culturel du pays et du raffinement des loisirs mondains. C'est pourquoi des auteurs dramatiques soulignent que leurs œuvres théâtrales entrent en compte dans la comparaison internationale et deviennent une source de fierté, non seulement pour eux-mêmes, mais aussi pour leurs compatriotes. Ainsi, Alexandre Soumarokov déclare en 1759 dans son périodique Trudolioubivaia Ptchela ${ }^{16}$ :

В то самое время, в которое возникъ, приведенъ и въ совершенство, в Россіи, Теятр твой, Мельпомена! всъ я преодольлъ трудности, всъ преодольл препятствія. На конецъ видите вы любезныя мои согражданя, что ни сочиненія мои ни Актеры вам стыда не приносятъ, и до чего в Германіи многими Стихотворцами не достигли, до того я одинъ, и въ такое еще время, въ которое у нас Науки словесныя только начинаются, и нашъ языкъ едва чиститься началъ, однимъ своимъ перомъ достигнуть могъ ${ }^{17}$.

C'est, entre autres choses, grâce au développement de son théâtre, que la Russie peut, d'après Soumarokov, soutenir "sans honte » la comparaison avec l'Allemagne, la France, l'Angleterre. Mais en le représentant, la presse française ne fait pas que rendre compte d'une réalité culturelle, elle propose un miroir au théâtre français - étranger mais non plus autre, mélange de proximité et de distance - et l'image qu'il lui renvoie est source d'admiration autant que d'inquiétudes.

Cette étude s'articulera en deux temps, et se proposera trois objectifs. Il s'agit d'abord de donner un aperçu de la représentation du théâtre russe dans certains périodiques qui circulaient en Europe. Grâce à un relevé d'articles qui portent sur notre sujet, nous proposerons des hypothèses quant à leur quantité, leur qualité et leur évolution au cours de la période (1755-1777). Puis, dans le détail du texte, nous remarquerons les problèmes posés par l'étude de la presse : ce genre appelle une analyse détaillée mais celle-ci est rendue complexe par les modalités d'énonciation. L'exposé d'une méthode qui consiste à faire se croiser les

\footnotetext{
${ }^{14}$ Voltaire, Anecdotes sur le czar Pierre le Grand ; Histoire de l'empire de Russie sous Pierre le Grand, édition de Michel Mervaud et Christiane Mervaud, avec la collaboration d'Andrew Brown et Ulla Kölving, Oxford, Voltaire Foundation, 1999.

${ }^{15}$ Derek Offord et Vladislav Rjeoutski, «Translation and propaganda in the mid-eighteenth century: French versions of Sumarokov's tragedy Sinav and Truvor », 2013 : https://frinru.ilrt.bris.ac.uk/introduction/translationand-propaganda-mid-eighteenth-century-french-versions-sumarokov\%E2\%80\%9gs-tragedy\# ednrefi2 (dernier accès: 07.05.15). Les auteurs citent Rude and Barbarous Kingdom: Russia in the Accounts of the Sixteenth-Century English Voyagers, ed. Lloyd E. Berry and Robert O. Crummey, Madison, University of Wisconsin Press, 1968 et Michel Mervaud et Jean-Claude Roberti, Une infinie brutalité. L'image de la Russie dans la France des XVIe et XVIIe siècles [An Infinite Brutality. The Image of Russia in Sixteenth- and Seventeenth-Century France], Paris, IMSECO-Institut d'études slaves, 1991.

${ }^{16}$ Alexandre Soumarokov, Трудолюбивая пчела [L’Abeille industrieuse], Saint-Pétersbourg, Izdatelstvo Akademii Naouk, 1759 .

${ }^{17}$ Ibid., « О копистах » [« Des copistes »] (décembre 1759) : «Au moment même où ton théâtre, Melpomène, arrive à la perfection en Russie ! J'ai surmonté toutes les difficultés, j’ai surmonté tous les obstacles. A la fin vous verrez, mes chers concitoyens, que vous n'aurez pas à avoir honte ni de mes œuvres ni de mes acteurs, et que seul je suis arrivé à faire ce que peu de dramaturges ont accompli, en Allemagne, et encore à un moment où les belleslettres naissaient tout juste chez nous, et où notre langue commençait juste à s'épurer, seul j’y suis parvenu, grâce à ma plume. »
} 
techniques d'analyse de la presse et du genre dramatique sera aussi l'occasion de souligner les spécificités de la représentation du théâtre russe.

\section{Exposition}

Commençons par proposer quelques pistes d'analyse de la représentation du théâtre russe dans la presse francophone européenne au cours de la seconde moitié du XVIII ${ }^{\mathrm{e}}$ siècle. Cette approche s'inscrit naturellement dans une observation plus générale de la représentation de la Russie dans les journaux. Nous ne développerons pas ce sujet ici, mais force est de constater une présence croissante des « sujets russes ». En revanche, avant d'aborder la question du théâtre, il est nécessaire de souligner la proportion minime de sujets russes qui portent sur les belles-lettres, par rapport aux sujets que les rédacteurs placent dans la catégorie des sciences : découvertes, géographie, récits d'explorateurs ${ }^{18}$, histoire politique. Il est difficile de procéder à une analyse exhaustive de l'ensemble des périodiques français ou francophones pour recenser l'ensemble des mentions au théâtre russe. En effet, les publications périodiques francophones en Europe sont beaucoup plus nombreuses et variées qu'en Russie, par exemple. La tradition périodique est plus ancienne, comme l'expose le Dictionnaire des journaux de Jean Sgard qui recouvre une période de près de deux siècles ${ }^{19}$. Une première approche peut cependant être proposée grâce à l'analyse d'un nombre réduit d'articles choisis dans des périodiques dont on sait qu'ils permettaient la circulation des idées et des représentations en Europe. Ainsi, L'Esprit des journaux, somme de journaux français et étrangers publiée de 1772 à 1818 et appartenant à la catégorie des "anthologies de presse ${ }^{20}$ ", avait pour mission de faire circuler l'information culturelle et scientifique à l'échelle la plus large, ainsi que le démontrent les chercheurs du Groupe d'Étude du Dix-Huitième Siècle et des Révolutions (GEDHSR) de l'université de Liège, qui ont mené un projet d'édition et d'exploitation des ressources du périodique ${ }^{21}$. Certes, comme le rappelle Alexandre Stroev, « le choix arbitraire et le caractère disparate des textes interdisent des généralisations ». Cependant, « on ne peut pas ignorer une certaine cohérence de publications qui permet de dégager quelques tendances majeures ${ }^{22}{ }^{2}$. Ainsi, les stratégies de publication, notamment des comptes rendus sur le théâtre, mais aussi du développement de la critique théâtrale comme un indicateur de développement culturel, sont représentées dans le périodique. En effet, rappelle Bernard Rentier, " quand L'Esprit des journaux ne volait pas, purement et simplement, des articles à des concurrents, il donnait aux uns et aux autres un supplément de caisse de résonance dans le débat public ${ }^{23}$." L'Esprit des Journaux constitue donc un bon point de départ pour observer une perception générale des littératures européennes, des publications et des sujets à la mode sur la période qu'il recouvre. On peut également se référer aux sources des articles de ce périodique : la Gazette universelle de littérature, ou Gazette des Deux-Ponts, publiée aux

\footnotetext{
${ }^{18}$ Voir notre contribution au colloque international L'invention de la Sibérie, (novembre 2010, Université Lyon II ISH) qui détaille la critique dans la presse de quelques « relations savantes » des explorateurs français en Russie. Carole Chapin, "Images de la Sibérie vue par la presse française et russe du XVIII siècle ", L’invention de la Sibérie par les voyageurs et écrivains français (XVIII ${ }^{e}-X I X^{e}$ siècles), sous la dir. d'Alexandre Stroev et Sarga Moussa, Paris, Institut d'études slaves, 2014, p. 100-110.

${ }^{19}$ Dictionnaire des journaux : 1600-1789, sous la dir. de Jean Sgard, Paris, Universitas, 1991.

${ }^{20} \mathrm{Ibid}$., t. II, « Répartition et typologie des titres », p. 1131-1140.

${ }^{21}$ http://web.philo.ulg.ac.be/gedhsr/index-annees-1772-1789a/ (dernier accès : octobre 2015).

${ }^{22}$ Alexandre Stroev, « La Russie dans L'Esprit des journaux », in Daniel Droixhe (dir.), L'Esprit des journaux : un périodique européen au XVIII siècle, Bruxelles, Le Cri, 2010, p. 263.

${ }^{23}$ Allocution d'ouverture du colloque « Diffusion et transferts de la modernité dans L'Esprit des journaux », les 16 et 17 février 2009 .
} 
Deux-Ponts de 1770 à 1777 par Joseph-Gaspard Dubois-Fontanelle, par exemple, catalogue critique des nouvelles parutions européennes, est souvent reprise, tout comme des articles de L'Année littéraire, d'Elie-Catherine Fréron, publié de 1754 à $1776^{24}$. Le Journal étranger (17541762), créé par Ignace de la Marche-Courmont, aux nombreux rédacteurs successifs tels que Grimm, Prévost, Fréron, qui bénéficient souvent d'une certaine notoriété, se trouvait également au cœur d'un réseau international, et affichait une ligne éditoriale nette: contribuer à la diffusion des publications littéraires étrangères ${ }^{25}$. Pour cette étude, nous utilisons cette série non exhaustive de périodiques français et francophones.

Le Journal étranger mentionne la Russie onze fois entre 1755 et 1762, presqu'une fois par numéro, alors même que l'« Avertissement » de $1756^{26}$ signale qu'il ne mentionnera plus que les pays « importants », c'est-à-dire, d'après lui, l'Allemagne, l'Italie, l'Angleterre, l'Espagne et le Portugal. Pourtant, si la Russie n'est pas encore comptée au nombre des nations importantes du point de vue des belles-lettres, elle occupe, en pleine guerre de Sept Ans, une place majeure sur la scène militaire et politique : toute action en sa faveur, y compris lui faire une place dans les pages du journal, peut apporter un bénéfice au-delà du plaisir du lecteur. Sur ces onze mentions, neuf sont scientifiques (signalant des découvertes ou des explorations), et deux seulement littéraires, dont celle que nous utiliserons sur le théâtre ${ }^{27}$. Ainsi, les changements de rédacteurs perpétuent malgré tout une forme de continuité et un réseau journalistique important. En 1758, par exemple, le Journal étranger publie un article intitulé « Discours de M. Rahout prononcé devant une assemblée de l'université impériale ${ }^{28}$ », identique à celui paru deux mois plus tôt dans l'Année littéraire de Fréron ${ }^{29}$. Certes, à cette date, ce dernier a cédé sa place à Anne Gabriel Meusnieur de Querlon, mais les deux périodiques restent liés par la présence de ce même article, qui témoigne, si ce n'est de la proximité évidente des rédacteurs, au moins de l'existence de réseaux ou d'intérêts communs. Avant 1776, la Gazette universelle de littérature, si l'on excepte le cas problématique du "Théâtre du prince Clénerzow ${ }^{30}$ ", œuvre du dramaturge français Louis de Carmontelle masqué par la fausse identité d'un prince de Russie, ne mentionne pas le théâtre russe. On trouve pourtant un nombre important de textes sur la Russie dans ses pages dès les premières publications : quelques textes d'histoire, et une majorité de récits de voyage à travers la Russie, notamment en Sibérie. Cette gazette est une des sources privilégiées de l'Esprit des journaux, qui reprend tous ses articles sur le théâtre russe. Si l'intérêt de l'Esprit des journaux pour le théâtre étranger est connu ${ }^{31}$, ceci n'en reste pas moins surprenant, car la reprise est loin d'être systématique dans le cas du théâtre allemand, encore moins dans celui du théâtre anglais, qui est pourtant le plus représenté dans la gazette.

\footnotetext{
${ }^{24}$ Elie-Catherine Fréron, L’Année littéraire, Lambert, 1754-64.

${ }^{25}$ L'objet n'étant pas la présentation précise de ces journaux, nous nous bornons à une très brève présentation de chacun d'entre eux, et nous renvoyons le lecteur aux ouvrages de référence, entre autres: Jean Sgard (dir.), Dictionnaire des journaux 16oo-1789, Paris, Universitas, 1991, ressources et articles sur l'Esprit des journaux, par le Groupe d'Étude du Dix-Huitième Siècle et des Révolutions (GEDHSR) de l'Université de Liège :

http://web.philo.ulg.ac.be/gedhsr/lesprit-des-journaux/ (dernier accès : 07.05.15).

${ }^{26}$ Peu après la succession, en juillet 1755 , de Fréron à Prévost.

${ }^{27}$ Abbé Prévost, « Sinave et Trouvore, tragédie russienne, en vers, par M. Soumarokoff », Journal étranger, Paris et Bruxelles, Durant, avril 1755 .

${ }^{28}$ Anne Gabriel Meusnieur de Querlon, « Discours de M. Rahout prononcé devant une assemblée de l'université impériale », Journal étranger, Paris et Bruxelles, Durant, juin 1758.

29 Elie-Catherine Fréron, «Discours prononcé dans une assemblée de l'université impériale de Moscow », L’Année littéraire, Lambert, avril 1758.

${ }^{30}$ Voir ci-dessous, note 40.

${ }^{31}$ Le travail d'indexation de l'équipe de Liège met parfaitement en valeur l'intérêt de ce périodique pour les sujets sur le théâtre : http://www.gedhs.ulg.ac.be/recherches/espritdesjournaux/index.html (dernier accès le o7.05.15).
} 
On peut essayer d'établir une typologie de ces articles. Tous ne portent pas exclusivement sur le théâtre russe, ou sur un ouvrage qui en traite, mais ne le mentionnent qu' « en passant ", comme une illustration secondaire pour un sujet différent. On distinguera donc :

- des articles généraux sur la Russie ou la littérature russe, dont une partie est consacrée à son théâtre: le «Discours de M. Rahout prononcé devant une assemblée de l'université impériale », et un article intitulé "Lettre d'un jeune seigneur russe à M. de ** » paru dans l'Année Littéraire en $1760^{32}$;

- un article véritablement consacré au théâtre russe, par l'intermédiaire de la critique d'un ouvrage qui en retrace l'histoire, l' « Essai sur l'ancien théâtre russe » publié dans la Gazette universelle de Littérature et dans l'Esprit des journaux en $1776^{33}$;

- des articles précis sur des pièces russes qui en font une présentation approfondie, dans la tradition de la critique littéraire : "Sinave et Trouvore, tragédie russienne, en vers, par M. Soumarokoff ${ }^{34}$ », ou un compte rendu plus bref : " Rossei w' Archipelaghie, \&c. Les Russes dans l'Archipel, drame en trois actes et en vers alexandrins, par Monsieur Paul Potemkine, Saint-Pétersbourg, $1772^{35}$ », « Wospitanie, Komedia w'piati. Le Conte, comédie en cinq actes. A Pétersbourg, $1774^{36}$ ", et «Dobrodjeteli Uwents chamaja Wjernortiju, \&c. La Vertu couronnée par la fidélité. Comédie en 3 actes ; par Monsieur Michaila PRAKUDIN, officier des gardes, et membre de la société libre russe à l'université impériale de Moscow. A Moscow, $1775^{37} \gg$;

- enfin, le cas limite du « Théâtre du prince Clénerzow, russe, traduit en français par le baron de Bléning, Saxon ${ }^{38} »$. Il ne s'agit pas d'un dramaturge russe mais d'une identité empruntée par un auteur français, Carmontelle, pour publier son théâtre de société. Le traitement de ce changement d'identité par les journalistes peut néanmoins nous donner une indication sur la perception de la Russie et son traitement dans la presse.

À l'exception notable, donc, du développement sur la pièce de Soumarokov Sinav et Trouvor dans le Journal étranger de 1755, ces remarques préliminaires nous permettent déjà de

${ }^{32}$ Elie-Catherine Fréron, op.cit., « Lettre d'un jeune seigneur russe à M. de ** » (décembre 176o).

${ }^{33}$ Joseph-Gaspard Dubois-Fontanelle, "Essai sur l'ancien théâtre russe », Gazette universelle de littérature, aux Deux-Ponts, Paris : Lacombe, février 1776. Abbé Oudin et Louis-François de Lignac, « Essai sur l'ancien théâtre russe », L'Esprit des journaux, par une société de gens de lettres, Liège, Bruxelles, Paris : J.J. Tutot, mai 1776.

${ }^{34}$ Abbé Prévost, op.cit., « Sinave et Trouvore, tragédie russienne, en vers, par M. Soumarokoff » (avril 1755).

${ }^{35}$ Joseph-Gaspard Dubois-Fontanelle, op.cit., « Rossei w' Archipelaghie, \&c. Les Russes dans l'Archipel, drame en trois actes et en vers alexandrins, par Monsieur Paul Potemkine, Saint-Pétersbourg, 1772 » (mai 1776). Abbé Oudin et Louis-François de Lignac, op.cit., « Rossei w' Archipelaghie, \&c. Les Russes dans l'Archipel, drame en trois actes et en vers alexandrins, par Monsieur Paul Potemkine, Saint-Pétersbourg, $1772 »$ (janvier 1777).

${ }^{36}$ Joseph-Gaspard Dubois-Fontanelle, op.cit., «Wospitanie, Komedia w'piati (sic). Le Comte, comédie en cinq actes. A Pétersbourg, 1774 », novembre 1776. Abbé Oudin et Louis-François de Lignac, op.cit., «Wospitanie, Komedia w'piati. Le Conte, comédie en cinq actes. A Pétersbourg, 1774 », février 1777.

${ }^{37}$ Joseph-Gaspard Dubois-Fontanelle, op.cit., «Dobrodjeteli Uwents chamaja Wjernortiju (sic), \&c. La Vertu couronnée par la fidélité. Comédie en 3 actes ; par Monsieur Michaila PRAKUDIN, officier des gardes, et membre de la société libre russe à l'université impériale de Moscow. A Moscow, 1775 ", 1776. Abbé Oudin et Louis-François de Lignac, op.cit., « Dobrodjeteli Uwents chamaja Wjernortiju (sic), \&c. La Vertu couronnée par la fidélité. Comédie en 3 actes; par Monsieur Michaila PRAKUDIN, officier des gardes, et membre de la société libre russe à l'université impériale de Moscow. A Moscow, 1775 » (1777). Dans les deux cas, la translittération en français par les périodiques est fautive et quasi incompréhensible. La pièce dont il est question est une pièce de 1774 de Mikhaïl Ivanovitch Prokoudin-Gorsky / Михаил Иванович Прокудин-Горский (jusqu'en 1791, on trouve le nom d'auteur également sous la forme Prokoudin ou Prakoudin / Прокудин, Пракудин), intitulée Добродетель, увенчанная верностью [La vertu couronnée de la fidélité].

${ }^{8}$ Elie-Catherine Fréron, op.cit., «Théâtre du prince Clénerzow, russe, traduit en français par le baron de Bléning, Saxon » (1771). Joseph-Gaspard Dubois-Fontanelle, op.cit., « Théâtre du prince Clénerzow, russe, traduit en français par le baron de Bléning, Saxon » (1771). 
constater une évolution chronologique de l'intérêt et du registre de ces textes : non seulement le théâtre devient le centre des articles, mais les pièces mentionnées ne sont plus seulement des illustrations ou des faire valoir, elles tendent à être étudiées pour elles-mêmes, pour le divertissement qu'elles procurent et pour leurs qualités littéraires. Les raisons de cette évolution se trouvent dans les articles mêmes. Loin de signaler un déclin de l'intérêt pour les explorations scientifiques sur les territoires russes, qui serait la preuve d'une considération plus superficielle, elle signale au contraire une appréciation de plus en plus positive qui prend en compte son statut culturel : "La littérature russe devient un objet aussi intéressant pour nous que l'histoire de ce vaste empire ; et cependant l'une n'est guère plus connue que l'autre, du moins dans le midi de l'Europe », nous signalent en introduction les critiques du drame Les Russes dans l'Archipel de Michael Potemkine en 1776 et 1777. C'est grâce au théâtre que le peuple russe devient éduqué et raffiné, sous entendent la plupart de nos articles, à l'instar des pays préférés habituellement dans les périodiques. Sa littérature peut ainsi trouver grâce aux yeux des journalistes et de leurs lecteurs :

Avec de la religion, des mœurs, du patriotisme et des talents, il faut aussi avoir du monde et des manières ; la cour est l'école des personnes faites pour ces deux derniers objets, on a cru que les spectacles feraient le même effet pour l'enfance et la jeunesse, et d'après ce principe, le théâtre est devenu une partie de l'éducation qu'on donne en Russie ${ }^{39}$.

Le théâtre est présenté comme le signe de l'évolution d'un peuple. La langue russe, quant à elle, bénéficie d'une aura nouvelle :

Votre langue, pleine d'énergie et de délicatesse, au jugement de ceux qui la connaissent, n'est point renfermée dans les bornes d'un Etat resserré ; elle embrasse l'Europe et l'Asie, elle est le lien de cent peuples divers, soumis à la domination de ce vaste empire. Elle est également propre aux sciences abstraites, au sublime et aux grâces de la poésie : bientôt toute la littérature sera de son ressort. Elle traite avec succès le genre dramatique ; vous avez des Tragédies intéressantes, et dès les premiers pas que votre nation fait dans cette carrière, elle déploie la force du génie ${ }^{40}$

Ce type d'appréciation vise à faire disparaître progressivement le soupçon de barbarie, et le russe, loin d'être un obstacle à la création poétique et littéraire, peut produire les formes artistiques en vogue dans toute l'Europe. En somme, l'apparition du théâtre russe dans l'espace des périodiques européens qui en révèlent l'existence et en assurent la diffusion, contribue à donner à la Russie, pour l'opinion publique, une légitimité culturelle.

\section{L'évolution des représentations et le rôle des périodiques}

Le Journal Etranger en tire une gloire particulière de son rôle dans la diversification des échanges à l'échelle internationale :

Il faudrait gémir de la décadence des lettres, ou déplorer ouvertement la perte du goût, si l'on pouvait supposer que toute l'Europe n'eût point applaudi au plus beau dessein qu'on eût

\footnotetext{
${ }^{39}$ Joseph-Gaspard Dubois-Fontanelle, op.cit., «Essai sur l'ancien théâtre russe » (février 1776). Abbé Oudin et Louis-François de Lignac, op.cit., « Essai sur l’ancien théâtre russe » (mai 1776).

${ }^{40}$ Elie-Catherine Fréron, op.cit., «Discours prononcé dans une assemblée de l'université impériale de Moscow » (avril 1758). Anne Gabriel Meusnieur de Querlon, op.cit., « Discours de M. Rahout prononcé devant une assemblée de l'université impériale » (juin 1758).
} 
jamais formé pour la communication des lumières humaines, et par conséquent pour le progrès général des sciences et des arts ${ }^{41}$.

C'est pourquoi la position des hommes de lettres russes est saluée par les hommes de lettres français, ce que souligne Fréron en rapportant le discours de Rahout: "Contents de vos propres richesses, vous pourriez, Messieurs, négliger des secours étrangers ${ }^{42}$ », car ces derniers, au contraire, ne répugnent pas à l'emprunt et à l'adaptation pour la constitution de leur théâtre national :

Il faudrait être bien injuste pour en refuser à M. Soumarokoff, parce qu'il a su estimer et mettre à profit des beautés étrangères. Une ignorance honteuse ne met que trop d'auteurs modernes à l'abri de ce reproche. Réduits à piller leurs contemporains et leurs compatriotes, on ne voit dans tous leurs ouvrages que des larcins grossiers, au lieu d'une savante imitation ${ }^{43}$.

Le rapport à l'imitation, à l'emprunt et aux adaptations est en effet un sujet récurrent des périodiques. Cependant, le soutien apporté par les journaux européens va principalement à la posture modérée prônée par la cour : il s'agit d'emprunter avec caution, d'adapter au contexte russe, d'encourager les traductions, d'afficher une volonté de transmission sans dévaloriser les productions nationales, de les encourager. Les autres voies de communication entre lettrés de l'Europe, proches de ces positions officielles, comme l'Académie des sciences, les rencontres diplomatiques, favorisent la transmission de ce discours, plus particulièrement.

La publication, dans des journaux étrangers, des idées qui servent la stratégie de politique culturelle du pouvoir russe, soutient cette même stratégie et peut être utilisée en retour, en Russie, pour en prouver la légitimité et l'universalité. Cependant, les journaux sont caractérisés par leur polyphonie et on peut y déceler les ambiguïtés d'un discours qui sert les intérêts russes sans cesser de porter la supériorité nationale. Par exemple, l'éloge de Soumarokov par Andreï Chouvalov dans le Journal étranger, cité ci-dessus, fait écho à celui de L'Année Littéraire, qui glisse que l'auteur est " privé d'un génie créateur ${ }^{44}$ » et qu'il doit aller puiser ses sources en France. Il se heurte néanmoins à l'obstacle de la définition du genre journalistique, caractérisé par sa polyphonie. C'est seulement par une étude systématique et détaillée de la poétique des textes que l'on peut éviter cet écueil. Nous proposons donc de suivre la métaphore fréquemment utilisée par les journaux - celle du théâtre - pour appliquer les méthodes de la poétique du genre dramatique dans le cadre de ces analyses sur la pensée théâtrale.

Le journal est une scène, sur laquelle a lieu la représentation d'une réalité qui peut emprunter les voies du comique et du tragique. Cette représentation a lieu dans un espace restreint, celui de l'article : elle est nécessairement transformée, qu'elle cherche à transformer le réel ou à le dépeindre de la façon la plus vraisemblable possible. Le metteur en scène de cette réalité est le journaliste, dépositaire d'une pluralité de voix. Leur identification s'inscrit dans un jeu de masques et de triple énonciation. Il faut par exemple essayer de comprendre, dans la louange de Soumarokov que nous venons de citer, le message implicite qui complète la critique de Sinav et Trouvor. Après l'analyse détaillée des beautés de l'écriture du dramaturge, la fin de l'article vient révéler que ces beautés ne sont pas originales, mais empruntées : « Il

\footnotetext{
${ }^{41}$ Abbé Prévost, op.cit., « Avertissement de M. l'abbé Prévost sur la continuation du Journal Étranger » (janvier 1755).

${ }^{42}$ Elie-Catherine Fréron, op.cit.

${ }^{43}$ Abbé Prévost, op.cit., « Sinave et Trouvore, tragédie russienne, en vers, par M. Soumarokoff » (avril 1755).

${ }^{44}$ Elie-Catherine Fréron, op.cit., « Lettre d'un jeune seigneur russe à M. de ** », décembre 1760.
} 
semble, au reste, que M. Soumarokov avait acquis la connaissance de quelques théâtres étrangers, avant que d'enrichir de cette tragédie celui de sa nation ${ }^{45}$. » On remarque une ressemblance frappante entre la louange du Journal étranger, et celle reprise par l'Année littéraire sur ce même auteur : « il sait imiter avec adresse ; incapable d'atteindre à Corneille, il a choisi Racine pour modèle ; la vivacité de son esprit a suppléé à la sécheresse de son imagination ${ }^{46}$. » La mise en scène de l'espace de l'article permet de maintenir une certaine ambiguïté : le rédacteur insiste sur la grandeur des modèles français, tout en remplissant les objectifs décrits dans l'avertissement du périodique, c'est à dire de jouer un rôle de diffusion et de promotion des belles-lettres étrangères, ici la littérature russe. C'est d'ailleurs ce qui intéresse tout particulièrement Soumarokov, montrant ainsi les retombées possibles de ces publications étrangères sur son image en Russie et sur la vie littéraire russe. Ainsi, dans le même article que nous citions en introduction, le dramaturge se flatte de voir son nom circuler dans les journaux français et allemands ${ }^{47}$, et Derek Offord et Vladislav Rjéoutski ont analysé le rôle important que pouvait jouer la circulation des œuvres et du nom de Soumarokov à l'étranger ${ }^{48}$.

En effet, le journal tient aussi lieu de tribune, aux stratégies énonciatives multiples. Le ou les message(s) sont portés par plusieurs voix : voix du journaliste, d'un personnage réel ou fictif, procédé de la citation et de la reprise, de l'actualité ponctuelle mais aussi du caractère périodique de publication des journaux. Si les articles s'inscrivent dans un contexte éphémère, les reprises successives et la circulation des feuillets leur permettent également un renouvellement incessant. Il faut donc s'attacher à identifier ses destinataires, là où, comme au théâtre, ils étaient l'enjeu d'un divertissement, la cible d'une visée didactique et morale, qui s’inscrit parfois dans des enjeux plus larges. L'article publié par Fréron en décembre 176o, « Lettre d'un jeune seigneur russe à M. de ** », plus encore que celui du Journal étranger, illustre les ambiguïtés de ces stratégies de communication et la raison pour laquelle le genre du journal périodique les sert particulièrement efficacement. On assiste alors à une véritable mise en scène de la superposition des voix : Fréron a demandé des nouvelles à un de ses amis pour pouvoir informer ses lecteurs de l'état de la littérature en Russie. Suite à cette demande, prétendument spontanée, Fréron publie la lettre de réponse, qui elle-même rapporte un discours prononcé par un troisième personnage. Les informations sur les réseaux relationnels des journalistes, hommes de lettres et diplomates, et le fait que le chevalier d'Éon, diplomate français à Saint-Pétersbourg, soit l'ami mentionné par Fréron ${ }^{49}$, montrent que la démarche de publication d'un article sur l'état de la littérature russe n'est pas spontanée mais le fruit d'une stratégie concertée entre les deux « amis », qui y trouvent chacun leur intérêt. Quant au troisième personnage, qui derrière l'anonymat prend les traits du comte Andreï Chouvalov, il avait également tout intérêt à prendre part à la concertation, également favorable à l'image de la Russie en France et aux relations entre les deux pays. Fréron précise le contexte de publication : " La lettre que je vous envoie, Monsieur, sur les deux poètes les plus célèbres de la Russie, est de la composition de Monsieur le comte A.S, un de ces jeunes seigneurs dont je

\footnotetext{
${ }^{45}$ Abbé Prévost, op.cit., « Sinave et Trouvore, tragédie russienne, en vers, par M. Soumarokoff » (avril 1755).

${ }^{46}$ Elie-Catherine Fréron, op.cit., « Lettre d'un jeune seigneur russe à M. de ** », décembre 1760 .

${ }^{47}$ Alexandre Soumarokov, Трудолюбивая пчела [L’Abeille industrieuse], Saint-Pétersbourg, Izdatelstvo Akademii Naouk, 1759, voir, ci-dessus, note 17.

${ }^{48}$ Derek Offord et Vladislav Rjeoutski, «Translation and propaganda », art.cit.

49 Voir A. Строев, «Защита и прославление России : история сотрудничества шевалье д’Эона и аббата Фрерона ", Французы в научной и интеллектуальной жизни России XVIII-XX вв. [Alexandre Stroev, "Le chevalier d'Éon, collaborateur de l'abbé Fréron : défense et illustration de la Russie », Les Français dans la vie intellectuelle et scientifique en Russie $\left(X V I I I^{e} X X^{e}\right)$ ], Moscou, Olma Media Group, 2010, p. 164-174.
} 
vous parle ${ }^{50} . »$ Ces initiales A.S. font référence à Andreï Chouvalov, dont on orthographiait souvent le nom, en français, Shuvalov ou Schuvaloff. Le jeune comte qui appartient à l'élite aristocratique lettrée, en partie formée en France, et qui reste en contact régulier avec l'Europe, correspond en effet au personnage décrit dans l'article. La description des belleslettres russes apparaît alors non plus tant comme le fruit d'un intérêt nouveau de la part des journalistes francophones, que comme le résultat d'une impulsion donnée par un réseau franco-russe. Alexandre Stroev analyse ainsi l'identité probable du chevalier d'Éon derrière le masque anonyme de l' «ami $»^{51}$. Il explique ainsi un aspect des stratégies de publication qui alimentent l'image d'une Russie éclairée dans la presse européenne. A partir de 1756, le chevalier d'Éon était diplomate français à Saint-Pétersbourg et collaborait régulièrement avec Fréron pour L'Année littéraire. Cette collaboration était profitable aussi bien aux deux hommes qu'aux cours de France et de Russie : l'intérêt français était de renforcer le parti "pro-français» à la cour de Russie, aussi était-il de bon ton que les Français présents s'intéressent à la Russie et publient des choses à ce sujet. Le chevalier, de son côté, gagnait la confiance de ses interlocuteurs russes en contribuant à la promotion des belles-lettres russes dans les journaux. Fréron y trouvait quant à lui un autre intérêt, puisque les informations fournies par le chevalier d'Éon lui permettaient de concurrencer le Journal Encyclopédique sur les « sujets russes », mais en s'en distinguant par des sources nouvelles et davantage portées sur les arts, les lettres, et la vie culturelle. La lettre de Chouvalov se situe au cœur de cette stratégie, comme le sujet du théâtre en général, ainsi que conclut Alexandre Stroev :

Les articles des journaux, transmis par le chevalier d'Éon, complétés, révisés, réécrits ou rédigés par l'abbé Fréron, visent des objectifs littéraires et politiques. La haute réputation des écrivains et des savants russes renforce celle de l'impératrice, protectrice des muses. Si la langue russe, riche et belle, permet de créer des chefs-d'œuvre, si l'histoire de la nation remonte à nuit des temps, si les arts et les sciences se développent rapidement, alors il n'y a pas lieu de considérer la Russie comme un pays sauvage et despotique ${ }^{52}$.

La complexité de ces réseaux et de leurs enjeux croisés a des conséquences sur le contenu des articles mais aussi sur le plan formel. De ce point de vue, la polyphonie des périodiques s'accorde particulièrement bien avec la stratégie de diplomatie culturelle. Les articles portent ainsi la trace d'une presse sous influence. En conséquence, les éléments soulignés dans les rares pièces de théâtre russes critiquées par les journaux francophones ouvrent sur des opportunités de mettre le théâtre en regard des théâtres étrangers, et de comparer son développement à ce qui est présenté comme son degré le plus accompli. L'article de Fréron attribue certes de nombreuses qualités à Sumarokov, mais qui sont toutes empruntées à Racine, de même que ses défauts. Il cite ainsi Chouvalov :

Le pathétique domine dans toutes ses pièces, le sentiment y règne, une douce harmonie les embellit. Mais on peut lui reprocher d'avoir copié les défauts de son modèle, et de l'avoir imité jusque dans sa faiblesse, en faisant de l'amour l'âme de ses tragédies, en les avilissant par de petites intrigues ${ }^{53}$.

Cependant, il ne faut pas oublier que le rédacteur, comme le dramaturge derrière son

\footnotetext{
${ }^{50}$ Elie-Catherine Fréron, op.cit., « Lettre d'un jeune seigneur russe à M. de ** », décembre 1760 .

${ }^{51}$ А. Строев, « Защита и прославление России », art. cit., p. 171.

${ }^{2}$ А. Строев, « Защита и прославление России », art. cit., p. 173.

${ }^{53}$ Elie-Catherine Fréron, op.cit., « Lettre d'un jeune seigneur russe à M. de ** », décembre 1760.
} 
personnage et son acteur, garde une voix qui est toujours présente, ne serait-ce que par le choix de la citation. La Gazette universelle de littérature, du fait de son appartenance à la catégorie des gazettes, adopte, dans la majorité des articles, un style plus propice à la posture d'objectivité. Seules quelques phrases doivent précéder ou succéder au compte-rendu d'une pièce de théâtre pour engager ou non le spectateur à la voir ou à la lire. La notice de la pièce Vospitanie est menée selon les normes habituelles de la gazette : le récit de l'intrigue est encadré par des commentaires sur le contenu et la composition de la comédie. Cependant le rédacteur cède à la tentation de transmettre les stéréotypes sur la Russie en rappelant que cette pièce a les mêmes défauts que « celles que produit d'ordinaire l'enfance de l'Art ». Le préambule qui précède le récit de l'intrigue, « une critique agréable, des mœurs modernes des russes » est plus développé qu'à l'accoutumée, et rappelle avec complaisance plus qu'avec sévérité que la gallomanie est très répandue en Russie :

En Russie, comme dans toutes les autres contrées de l'Europe, les jeunes gens se piquent d'être à la française. Ils imitent nos modes, prennent les airs de nos petits-maîtres, et jusqu'à leurs mœurs. Les auteurs dramatiques, chacun dans sa sphère, prêchent leurs compatriotes pour les prévenir de cette épidémie universelle. Ils revêtent un sot d'un habit français et livrent le sot à la risée publique : mais, soit que l'auteur ait outré son personnage, soit qu'il reste encore sous des dehors ridicules quelque chose d'aimable, en sortant de la comédie, tout le monde veut encore être français ${ }^{54}$.

Les lecteurs francophones, et surtout français, sont toutefois sommés par un autre article de la Gazette de ne pas mépriser le théâtre russe :

Nous avertirons les français qui seront tentés de rire de ces détails, de se ressouvenir des farces, des mystères, des sotties de leurs aïeux ; les Russes, il y a très peu d'années, applaudissaient encore à de semblables inepties ; mais ils ne se sont éclairés que tard ; et si au commencement de ce siècle ils étaient encore bien loin des autres Nations, peu de temps leur a suffi pour se mettre au pair ; et si l'on peut juger de ce qu'ils feront par ce qu'ils ont fait, ils les devanceront peut-être ${ }^{55}$.

Le sujet du développement du théâtre russe est en effet intimement lié, dans les articles dont nous disposons, au sujet de la cour de Russie. Les auteurs majeurs, présentés par l'intermédiaire de Fréron, sont les protégés de l'impératrice: " Ils [Lomonossov et Soumarokov] étaient tous les deux sans biens, et jouissent actuellement d'une fortune honnête par les bienfaits de l'Impératrice ${ }^{56}$.», et la tragédie de Soumarokov, qui a mérité son article dans le Journal étranger, est présentée comme un témoignage, non tant de la gloire du dramaturge, mais de la gloire de Pierre le Grand et d'Elisabeth Ire : « C'est un monument des progrès que les arts, introduits par Pierre le Grand, ont fait en Russie sous la protection de son auguste fille ${ }^{57}$ ». L'articulation entre un discours admiratif et la promotion des intérêts russes d'une part, et un discours qui rappelle la supériorité du théâtre français d'autre part, semble répondre à des intérêts plus politiques qu'esthétiques.

La stratégie de rapprochement semble fonctionner, aidée par les événements militaires. Pavel Potemkine, écrivain et militaire, est en position de glorifier les victoires russes contre la

\footnotetext{
${ }^{54}$ Joseph-Gaspard Dubois-Fontanelle, op.cit., « Wospitanie, Komedia w'piati. Le Conte, comédie en cinq actes. A Pétersbourg, 1774 » (novembre 1776).

${ }^{55}$ Joseph-Gaspard Dubois-Fontanelle, op.cit., « Essai sur l'ancien théâtre russe » (février 1776). Abbé Oudin et Louis-François de Lignac, op.cit., « Essai sur l'ancien théâtre russe » (mai 1776).

${ }^{56}$ Elie-Catherine Fréron, op.cit., « Lettre d'un jeune seigneur russe à M. de ** » (décembre 176o).

${ }^{57}$ Abbé Prévost, op.cit., « Sinave et Trouvore, tragédie russienne, en vers, par M. Soumarokoff » (avril 1755).
} 
Turquie pendant la dernière guerre. Publier sa pièce Les Russes dans l'archipel signifie, pour cette raison et aussi en raison des liens entre Catherine II et le parent éloigné de l'auteur le prince Grigori Potemkine, son nouveau favori, complaire à l'impératrice. Mais l'auteur de la critique prend toutes les précautions pour que l'image de la Russie se détache progressivement d'un exotisme teinté de barbarie, ou, tout du moins, de naïveté, pour être traitée comme l'égale des nations européennes éclairées :

Ce drame, dont nous avons donné le canevas, fait un honneur infini à M. Potemkin ; et n'eût-il d'autre mérite que celui de peindre une nation que nous ne connaissons que très imparfaitement, il serait toujours fort estimable. Mais quoique le fond ne puisse guère flatter que les russes, le style et les détails peuvent offrir des beautés pour toutes les nations ${ }^{58}$.

Dans ce contexte, le traitement de la supercherie de Louis de Carmontelle, qui a pris le masque du prince Clénerzow pour souligner les défauts du théâtre français, est à relire. Le regard de l'étranger est un procédé habituel, au XVIII siècle, pour prendre de la distance et porter un jugement nouveau et souvent satirique sur une réalité connue. Souvent, sur le modèle des Lettres persanes ou de L'Ingénu, le personnage principal, malgré son bon sens, est lui-même éventuellement comique. Ici, le prince Clénerzow n'a aucun des attributs ridicules ou n'est pas caractérisé par la naïveté que l'on pourrait associer à son personnage. Celui-ci au contraire illustre la présence des hommes de lettres russes en France, l'intensification des échanges qui fait qu'il est possible de croire au portrait et au récit du prince :

Le prince Clénerzow, peu satisfait de toutes les réponses que ceux qui revenaient de France faisaient à ses questions, se détermine à connaître par lui-même ce peuple célèbre, loué, critiqué, envié à l'excès par les autres nations. Il arrive à Paris, où il se répand, trois années de suite, dans les meilleures compagnies. (...) L'esprit exempt des préjugés qui nous aveuglent, il fut frappé surtout du peu de vraisemblance de nos représentations théâtrales, de la singularité choquante de faire converser debout tant de personnages pendant 24 heures, de l'indécente familiarité des discours que tiennent en pleine compagnie les femmes de chambre et les valets; il vit que les véritables mœurs sont chez nous très différentes des mœurs dramatiques, et c'est ce qui lui donna l'idée d'en tracer dans sa langue des images plus fidèles ${ }^{59}$.

Bien que de nombreux indices, parsemés dès le début de ces articles, aident le lecteur à comprendre la supercherie, l'image de la Russie, incarnée par ce faux prince, est bien éloignée de celle des barbares vêtus de peaux de bêtes qui s'exprime dans les récits des explorateurs en Sibérie. Le rôle de miroir de la société et des mœurs du temps est sans cesse détourné afin de modifier les systèmes de représentations. Sous une forme plaisante, il peut chercher à piquer ou à louer, à provoquer une réaction affective, intellectuelle ou performative. Aussi est-il particulièrement intéressant de chercher à comprendre quelle image de l'étranger est diffusée par son intermédiaire. L'image de l'étranger a toujours une fonction: humoristique, normative, de modèle ou de faire valoir, comme c'est peut-être le cas de la citation précédente, ou comme veut le faire Carmontelle, qui prend le masque du prince Clénerzow pour souligner, à l'inverse, les défauts du public français : «Ces comédies sont précédées

\footnotetext{
${ }^{58}$ Joseph-Gaspard Dubois-Fontanelle, op.cit., « Rossei w' Archipelaghie, \&c. Les Russes dans l'Archipel, drame en trois actes et en vers alexandrins, par Monsieur Paul Potemkine, Saint-Pétersbourg, 1772 » (mai 1776). Abbé Oudin et Louis-François de Lignac, op.cit., « Rossei w' Archipelaghie, \&c. Les Russes dans l'Archipel, drame en trois actes et en vers alexandrins, par Monsieur Paul Potemkine, Saint-Pétersbourg, 1772 » (janvier 1777).

59 Elie-Catherine Fréron, op.cit., «Théâtre du prince Clénerzow, russe, traduit en français par le baron de Bléning, Saxon » (1771).
} 
d'une lettre critique sur les spectacles de Paris et en général sur le goût qui règne en France. » Le prince Clénerzow, nous dit-on, « ne savait pas que c'est la manie des petites loges qui prive le public d'entendre des nouveautés ${ }^{60} »$. Le journal prolonge la mystification et la mise en scène à sa guise : la Gazette de littérature démasque sans façons Carmontelle, quand Fréron préfère, jusqu'à la fin du passage, semer le doute sans le lever pour profiter encore du jeu de miroir :

Il vous paraitra sans doute assez extraordinaire, Monsieur, qu'un Étranger ait entrepris de dessiner avec des crayons russes le tableau si mobile de nos travers [...] mais, ce qui doit encore vous surprendre davantage, c'est que vous trouverez dans ce Théâtre une connaissance singulière des ridicules et du ton de nos sociétés ${ }^{61}$.

Le journal est donc à la fois une représentation du public et un guide pour un public futur. La critique dans le périodique est un aperçu de la réception du théâtre russe, mais influence aussi la réception et la perception des lecteurs qui sont eux-mêmes des spectateurs potentiels.

\section{Conclusion}

La représentation du théâtre russe est au cœur de l'attirance pour l'étranger qui caractérise la presse et la pensée théâtrale de la période. Mais la Russie n'est pas un pays comme les autres : c'est un miroir à la fois proche et lointain, inaccessible, incompréhensible mais que l'on peut chercher à s'approprier. Y a-t-il une véritable admiration pour le modèle russe en plein développement, comme le laisserait croire l'attitude du journaliste de la Gazette universelle de littérature? L'intermédiaire des sujets dramatiques, les jeux diplomatiques et le travail sur les représentations dans la presse auront conduit à un intérêt, voire à un " goût russe » en France et en Europe, où l'on ne se représente désormais plus les lettres russes comme l'enfance de l'art mais, peut-être, comme des futures rivales. La perception du théâtre russe dans la presse française informe le lecteur, tant hier qu'aujourd'hui, bien au-delà du discours critique sur les belles-lettres étrangères. L'analyse littéraire des sources, couplée à l'analyse des réseaux, permet également d'en savoir plus sur les relations et les stratégies diplomatiques du temps. La presse, tout comme le théâtre, était alors chargée d'une puissance symbolique et de communication qui pouvaient servir des intérêts politiques autant que personnels. Les nouveaux auteurs et lecteurs parvinrent progressivement à s'approprier ce nouveau moyen de communication qui nous est parvenu comme un précieux témoignage.

\footnotetext{
${ }^{60}$ Loc.cit.

${ }^{61}$ Loc.cit.
} 